\title{
The Effects of Epiretinal Membrane on the Treatment of Diabetic Macular Edema with Intravitreal Ranibizumab
}

\author{
(D) Yalçın Karaküçük, (i) Süleyman Okudan \\ Department of Ophthalmology, Selcuk University, Faculty of Medicine, Konya, Turkey
}

\begin{abstract}
Introduction: This study aimed to compare the response to ranibizumab therapy in naive diabetic macular edema (DME) patients with and without an epiretinal membrane (ERM).

Methods: A retrospective chart review was performed on 71 eyes in 71 DME patients. The patients were divided into a DME without ERM [ERM (-)] group ( $n=34)$ and DME with ERM [ERM (+)] group ( $n=37)$. Best-corrected visual acuity (BCVA), central macular thickness (CMT) and subfoveal choroidal thickness (SFCT) were evaluated.

Results: There were no significant differences in age or gender distribution between the patients in the ERM (-) and ERM (+) groups ( $p=0.052, p=0.96$, respectively). In both groups, CMT showed a significant decrease during the first, second and third months ( $p<0.01$ for each in both groups). Therefore, BCVA increased in both groups and made significant gains from the second month ( $p=0.044$ in the ERM (-) group and $p=0.006$ in the ERM (+) group). CMT and BCVA values did not differ significantly between the ERM (-) and ERM (+) groups in the baseline, first month, second month or third month. In the ERM (-) group, SFCT values decreased significantly as compared to baseline in the first month, second month and third month ( $p=0.001$, $p<0.001, p<0.001$, respectively). In the ERM (+) group, the first month SFCT value did not change significantly $(p=0.389)$, but it decreased significantly in the second month and third month as compared to baseline $(p<0.001, p<0.001$, respectively).

Discussion and Conclusion: Based on studying patients for three months, we determined that a series of three monthly injections of ranibizumab ensured a statistically significant improvement in visual acuity and a decrease in CMT and SFCT in diabetic patients with or without ERM.

Keywords: Diabetic macular edema; epiretinal membrane (ERM); ranibizumab.
\end{abstract}

$\mathrm{D}$ iabetic retinopathy and diabetic macular edema (DME) are the most common causes of visual deterioration in diabetic patients. Until the discovery of anti-vascular endothelial growth factor drugs (anti-VEGFs), laser photocoagulation was the standard treatment ${ }^{[1]}$. Today, the application of intravitreal anti-VEGFs is the most common and most effective first-choice treatment modality ${ }^{[2-5]}$. Many studies have shown that monthly intravitreal injections of ranibizumab (IVR, Lucentis; Genentech, Inc., South San Francisco, CA, USA) ensure a decrease in central foveal thickness and an increase in visual acuity in most of the patients with DME. However, some patients may show a poor response to anti-VEGFs, or in others, intravitreal anti-VEGF therapy alone may be insufficient in controlling $\mathrm{DME}^{[6-13]}$. According to some studies, the presence of epiretinal membrane (ERM), which is a disorder of the vitreomacular interface characterized by symptoms of decreased visual acuity and metamorphopsia, is one condition that affects the response to anti-VEGFs ${ }^{[10-14]}$. It should be noted that there is a higher prevalence of vitreomacular interface abnor-

Correspondence (iletişim): Yalçın Karaküçük, M.D. Selcuk Universitesi Tip Fakultesi, Goz Hastaliklari Anabilim Dali, Konya, Turkey Phone (Telefon): +90 5360652075 E-mail (E-posta): drkarakucuk83@gmail.com 
malities, including ERM, in diabetic patients, and thanks to the development of optical coherence tomography (OCT), these abnormalities can now be detected very easily ${ }^{[13-18]}$.

The present study aims to investigate the effects of ERM on visual acuity, central macular thickness (CMT) and subfoveal choroidal thickness (SFCT) changes in DME patients receiving intravitreal ranibizumab (IVR) therapy.

\section{Materials and Methods}

This work a retrospective comparative study that included patients who had undergone IVR therapy as a treatment for newly diagnosed DME at the Selcuk University Department of Ophthalmology between July 2017 and January 2019. Approval for the data collection and analysis was obtained from the ethics committee of the university (Protocol No: 2019/265), and all patients provided informed consent. The methodology of the study was conducted in accordance with the tenets of the Helsinki Declaration.

A retrospective comparative chart review was performed on 71 eyes in 71 patients. The patients were divided into a control group with only DME $(n=34)$ and a study group with DME and ERM $(n=37)$. Patients who received a series of three consecutive monthly doses of ranibizumab and were followed up for four months were included in this study. The inclusion criteria for the study was the presence of clinically significant DME based on a fundus examination, the presence of angiographically confirmed DME, the presence of DME and ERM documented by OCT and ultrasonography and a CMT $>300 \mu \mathrm{m}$ on OCT. Clinically significant DME was defined as the following: thickening of the retina at or within $500 \mu \mathrm{m}$ of the center of the macula, hard exudates at or within $500 \mu \mathrm{m}$ of the center of the macula if associated with thickening of the adjacent retina (not including residual hard exudates remaining after the disappearance of retinal thickening) or any zone(s) of retinal thickening 1 disc area or larger of which any part is within 1 disc diameter of the center of the macula[19]. Patients who had previously undergone vitreoretinal surgery or cataract surgery, had a loss of vision from causes other than DME and ERM, had prior intravitreal injections of bevacizumab, ranibizumab or steroids and/or laser treatments or a follow-up duration $<3$ months were excluded. All patients underwent a complete eye examination that included best-corrected visual acuity (BCVA) measurements (ETDRS chart), slit-lamp biomicroscopy, indirect ophthalmoscopy, IOP measurement using a Topcon model CT-800 (Topcon Corporation, Tokyo, Japan), fluorescein angiography (Visucam500, Carl Zeiss Meditec, Jena, Germany) and spectral-domain OCT
(Spectralis ${ }^{\circledast}$, Heidelberg Engineering, Heidelberg, Germany). The ERMs were recognizable as thin, hyperreflective bands anterior to the retina on OCT. The primary outcome measures were changes in CMT, SFCT, and BCVA.

\section{Injection Technique}

All injections were conducted under sterile conditions in an operating room. Eyes were anesthetized with topical $0.5 \%$ proparacaine hydrochloride, and $5 \%$ povidone-iodine was used for endophthalmitis prophylaxis. Subsequently, a 0.5-mg IVR injection (Lucentis; Novartis Pharma AG, Basel, Switzerland and Genentech, Inc., South San Francisco, CA, USA) was administered at the superotemporal pars plana (4 $\mathrm{mm}$ posterior to the limbus) using a 30-gauge needle. Topical $0.5 \%$ moxifloxacin eye drops were prescribed for use four times daily for one week after the injection. Followup examinations were scheduled for the first day, the first week and first, second and third months postoperatively. BCVA and OCT examinations were performed in the first-, second-, third- and fourth-month follow-ups.

\section{Statistical Analysis}

Statistical analysis was performed using SPSS version 15.0 (SPSS Inc., Chicago, IL, USA). A p-value of less than 0.05 was considered to be statistically significant. Mean, standard deviation, median, minimum and maximum value frequency and percentage were used as descriptive statistics. The distribution of the variables was checked using a Kolmogorov-Smirnov test. Mann-Whitney $U$ tests were used for the comparison of the quantitative data. Wilcoxon tests were used for repeated measurement analysis. A Chisquare test was used for the comparison of the qualitative data.

\section{Results}

There were no significant differences in the age or gender distribution between the ERM (-) and ERM (+) groups. Hypertension ratio, proliferative diabetic retinopathy ratio and diabetes mellitus duration were significantly higher in the ERM (+) group than in the ERM (-) group ( $p=0.039$, $\mathrm{p}=0.034, \mathrm{p}=0.001$, respectively) (Table 1 ).

In both groups, CMT showed a significant decrease in the first, second and third months. Again, BCVA increased in both groups and made significant gains from the second month. When the two groups were compared, CMT and $B C V A$ values did not differ significantly between the ERM $(-)$ and ERM (+) groups at the baseline, first month, second month or third month (Table 2). Initial SFCT was signifi- 
Table 1. Demographic and characteristic data of the ERM (-) group and ERM (+) group

\begin{tabular}{|c|c|c|c|c|c|c|c|}
\hline & \multicolumn{3}{|c|}{ ERM (-) } & \multicolumn{3}{|c|}{$\operatorname{ERM}(+)$} & \multirow[b]{2}{*}{$\mathbf{p}$} \\
\hline & Mean \pm SD & n (\%) & Median & Mean \pm SD & n (\%) & Median & \\
\hline Age (years) & $62.4 \pm 9.1$ & & 61.0 & $66.5 \pm 7.0$ & & 67.5 & $0.052^{m}$ \\
\hline \multicolumn{8}{|l|}{ Gender } \\
\hline Male & 18 & 58.1 & & 23 & 57.5 & & $0.962 \chi^{2}$ \\
\hline Female & 13 & 41.9 & & 17 & 42.5 & & \\
\hline \multicolumn{8}{|l|}{ HT } \\
\hline$(-)$ & 29 & 93.5 & & 30 & 75.0 & & $0.039 \chi^{2}$ \\
\hline$(+)$ & 2 & 6.5 & & 10 & 25.0 & & \\
\hline NPDR & 21 & 67.7 & & 17 & 42.5 & & $0.034 \chi^{2}$ \\
\hline PDR & 10 & 32.3 & & 23 & 57.5 & & \\
\hline Duration of DM (year) & $14.2 \pm 4.9$ & & 14.0 & $18.8 \pm 5.9$ & & 18.0 & $0.001^{m}$ \\
\hline
\end{tabular}

Table 2. Comparison of the parameter changes in the ERM (-) group and ERM (+) group

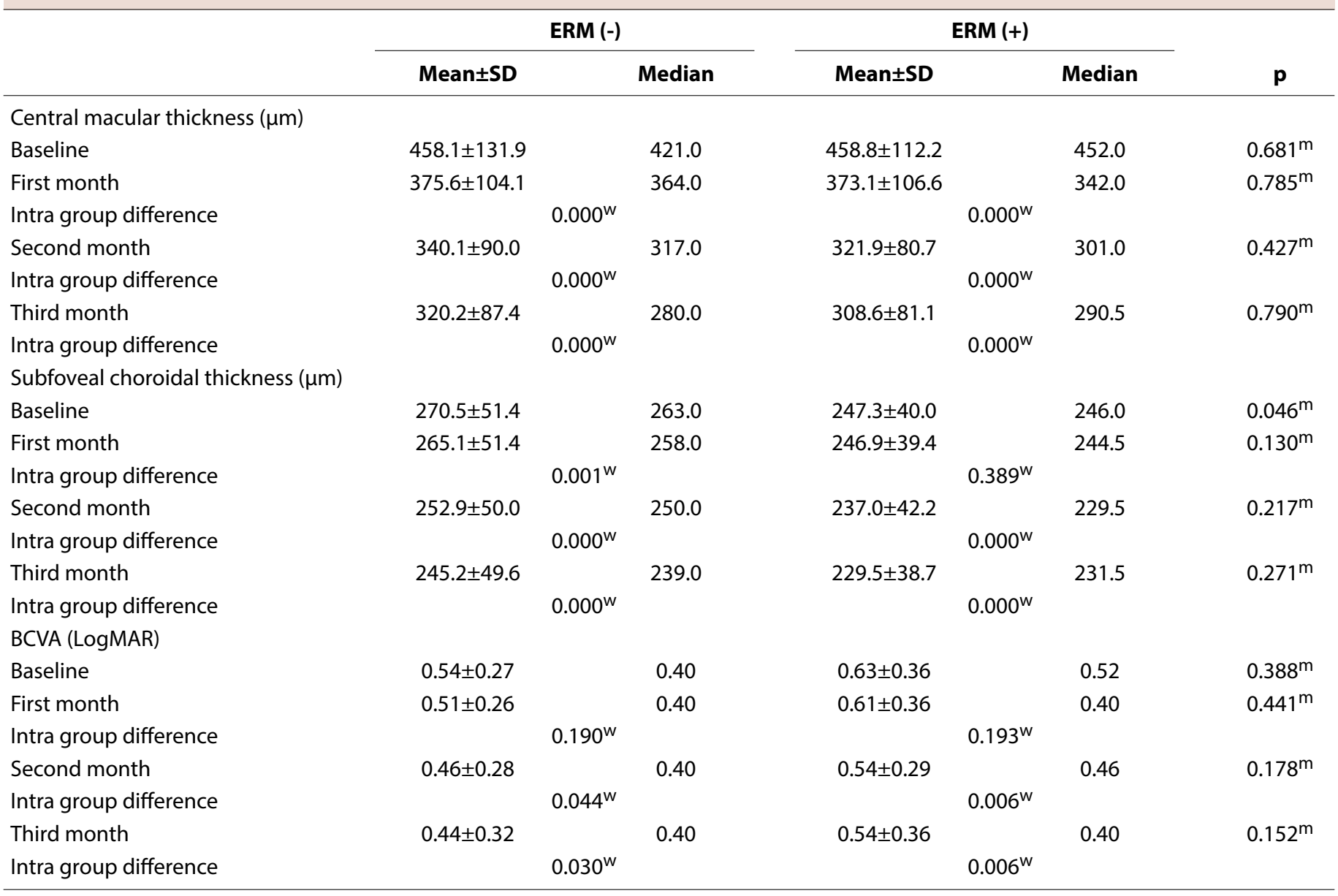

mMann-Whitney U test, wWilcoxon test, DME: Diabetic macular edema, ERM: Epiretinal membrane, BCVA: Best-corrected visual acuity

cantly higher in the ERM (+) group than in the ERM (-) group $(p=0.046)$. In the ERM (-) group, SFCT values decreased significantly $(p<0.05)$ compared to baseline at the first month, second month and third month. In the ERM (+) group, the SFCT value did not change significantly in the first month $(p=0.389)$, but it did decrease significantly in the second 


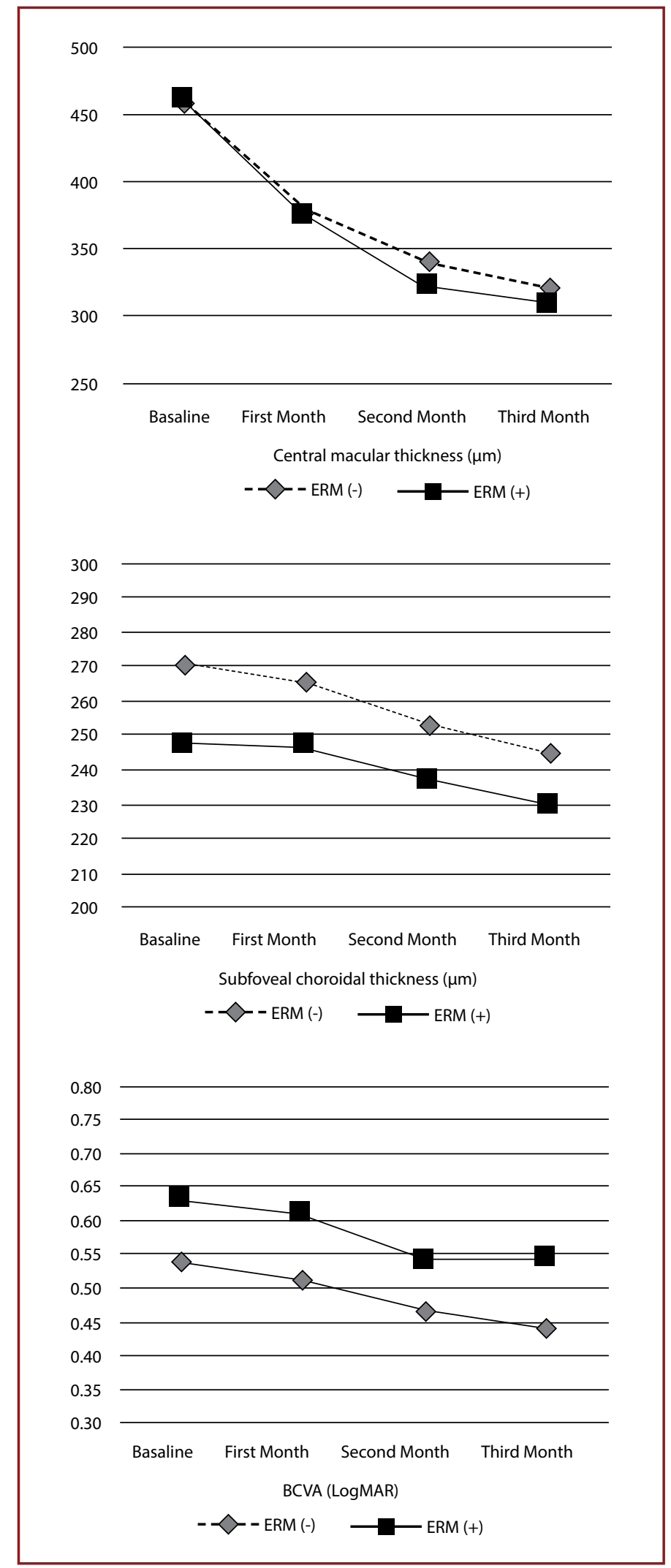

Figure 1. Graphs showing the changes in CMT $(\mu \mathrm{m})$, SFCT $(\mu \mathrm{m})$ and BCVA (LogMAR) during the 3-month follow up.

ERM: Epiretinal membrane, BCVA: Best-corrected visual acuity, SFCT: Subfoveal choroidal thickness month and third month as compared to baseline ( $p<0.01$, $p<0.01$, respectively) (Table 2). Figure 1 shows the changes in CMT, SFCT and BCVA during the three months.

\section{Discussion}

DME is the most common cause of visual impairment in diabetic patients. Today, the application of intravitreal anti-VEGFs is the first-choice and gold standard treatment modality. In this study, we determined that diabetic patients who had DME with ERM had a similar response to IVR concerning BCVA, CMT and SFCT as diabetic patients without ERM. ERM is one vitreomacular interface abnormality that may cause permanent visual loss by causing progressive retinal distortion ${ }^{[20]}$. ERM is more common in diabetic patients ${ }^{[11,21-23]}$. Kawasaki et al. ${ }^{[23]}$ found that older age and the presence of diabetes were the only two factors associated with ERM. Although the study and control groups in the current study were similar concerning age and gender, the values for hypertension and proliferative diabetic retinopathy were higher in the ERM (+) group, and the duration of diabetes was longer as well. This suggests that concomitant hypertension, diabetic retinopathy or the duration of diabetes mellitus may contribute to the formation of ERM. Whether the presence of ERM potentially affects the response to intravitreal antiVEGF treatments in DME patients is an important topic of this study.

Wong et al. ${ }^{[11]}$ compared the effects of IVR injection over a 1-year in diabetic patients with and without vitreoretinal interface abnormalities. They found significantly worse final central retinal thickness (CRT) in their ERM group after a mean of seven IVR injections.

Yoon et al. ${ }^{[12]}$ investigated the effects of the presence of vitreomacular interface abnormalities in patients with $D M E$ after three intravitreal anti-VEGF injections (either $0.3 \mathrm{mg}$ ranibizumab or $1.25 \mathrm{mg}$ bevacizumab). They found that diminished CRT and total macular volume were not statistically different between groups. They also determined that the highest increase in BCVA was in diabetic patients with a normal vitreomacular interface. This outcome shows that the presence of vitreomacular interface abnormalities has a negative effect on a visual prognosis. That the number of patients was so limited ( 15 eyes of 11 patients) and that all patients did not all receive the same anti-VEGF were major limitations of their study. There may be several reasons why the anti-VEGF response is poor in diabetic patients with ERM. The first hypothesis is that the additional structural damage to the photoreceptors arises from ERM may 
limit visual acuity improvements. The second is that ERM may decrease the effects of anti-VEGFs by preventing their penetration. In support of this second hypothesis, Namba et al. ${ }^{[24]}$ found reduced antibody permeability through an in vitro ERM model. In addition, the reason for the increase in retinal thickening in diabetic patients with ERM is related not only to rising vascular endothelial growth factor levels but also to anteroposterior or tangential traction. In support of this hypothesis, visual acuity significantly improves after surgical vitrectomy in diabetic patients with ERM ${ }^{[20]}$.

Kulikov et al. ${ }^{[13]}$ compared the effects of $0.3 \mathrm{mg}$ IVR in diabetic patients with normal and abnormal vitreoretinal interfaces. They found that CRT decreases to be statistically insignificant in all of their abnormal vitreoretinal interface subgroups (retinal wrinkling associated with eccentric ERM, ERM involving the macular center, vitreomacular adhesion and vitreomacular traction), whereas CRT showed a statistically significant decrease in patients with a normal vitreoretinal interface after a mean of 1.5 injections. In our study, we used three monthly ranibizumab $0.5 \mathrm{mg}$ injections and did not divide the patients with ERM into groups. Maryam et al. ${ }^{[9]}$ reported different outcomes that contradict other studies. They compared the effects of a singledose $2.5 \mathrm{mg} / 0.1 \mathrm{ml}$ intravitreal bevacizumab injection in diabetic patients with and without ERM. They found that the patients with ERM had a statistically significant improvement in visual acuity, while they also had a statistically insignificant decrease in CMT. Interestingly, in diabetic patients without ERM, the improvement in visual acuity was statistically insignificant and the decrease in CMT was statistically significant. In their study, baseline CMT was statistically similar between the groups, while baseline visual acuity in patients without ERM was statistically significantly better than in patients with ERM. The administration of a two-fold dose of intravitreal bevacizumab, a relatively short follow-up time (only 1 month) and having different baseline visual acuity values in the groups were important limitations of their study. In the present study, baseline values were similar concerning CMT and BCVA. CMT started to decrease in both groups from the first month, while the increase in BCVA was seen in both groups after the second injection. BCVA and CMT changes in the first three months were not statistically different in either group.

In the current study, SFCT began to decrease from the first month in both the ERM group and the non-ERM group, and the decrease continued after the third injection. The decrease in SFCT after three injections was not statistically significant when both groups were compared. In a previous study conducted by Cakır et al., 32 eyes in 27 patients with classic choroidal neovascularization who had undergone intravitreal $0.5 \mathrm{mg}$ ranibizumab injections with a minimum of a 2-year follow up were enrolled as the study group. They reported that long-term IVR treatment was associated with significant reductions in the ganglion cell layer and internal plexiform layer thicknesses ${ }^{[25]}$. In another study, SFCT seemed to decrease after intravitreal bevacizumab injections in eyes with neovascular age-related macular degeneration as compared to untreated eyes with the same condition ${ }^{[26]}$. Furthermore, SFCT decreased significantly within a short time in response to a single IVR injection in patients with branch and central retinal vein occlusion ${ }^{[27]}$. Differences in baseline values between the two groups concerning SFCT are among the limitations of our study.

The other limitations of the present study are that it was a retrospective study, the follow-up time was relatively short and the sample size was small. In addition, we did not divide the ERM cases into sub-groups.

In conclusion, our findings suggest that three consecutive monthly injections of ranibizumab ensured a statistically significant improvement in visual acuity and decreases in CMT and SFCT in diabetic patients either with or without ERM. Thus, ERM does not seem to affect the response of ranibizumab in patients with DME. Further studies that are randomized, controlled and prospective with a larger sample size are needed to confirm the results of our study.

Ethics Committee Approval: All procedures performed in this study involving human participants were in accordance with the ethical standards of the national research committee and with the 1964 Helsinki Declaration and its later amendments or comparable ethical standards.

Peer-review: Externally peer-reviewed.

Authorship Contributions: Concept: S.O., Y.K.; Design: S.O., Y.K.; Supervision: Y.K.; Funding: Y.K.; Materials: Y.K.; Data Collection or Processing: Y.K.; Analysis or Interpretation: Y.K.; Literature Search: Y.K.; Writing:Y.K.; Critical Review:Y.K., S.O.

Conflict of Interest: No potential conflicts of interest were reported by the authors.

\section{References}

1. Photocoagulation for diabetic macular edema. Early Treatment Diabetic Retinopathy Study report number 1. Early Treatment Diabetic Retinopathy Study research group. Arch Ophthalmol 1985;103:1796-806. [CrossRef]

2. Diabetic Retinopathy Clinical Research Network, Wells JA, Glassman AR, Ayala AR, Jampol LM, Aiello LP; et al. Aflibercept, bevacizumab, or ranibizumab for diabetic macular edema. $\mathrm{N}$ Engl J Med 2015;372:1193-203. [CrossRef] 
3. Ishibashi T, Li X, Koh A, Lai TY, Lee FL, Lee WK, et al; REVEAL Study Group. The REVEAL Study: Ranibizumab Monotherapy or Combined with Laser versus Laser Monotherapy in Asian Patients with Diabetic Macular Edema. Ophthalmology 2015; 122:1402-15. [CrossRef]

4. Nguyen QD, Brown DM, Marcus DM, Boyer DS, Patel S, Feiner L, et al; RISE and RIDE Research Group. Ranibizumab for diabetic macular edema: results from 2 phase III randomized trials: RISE and RIDE. Ophthalmology 2012;119:789-801. [CrossRef]

5. Mitchell P, Bandello F, Schmidt-Erfurth U, Lang GE, Massin P, Schlingemann RO, et al; RESTORE study group. The RESTORE study: ranibizumab monotherapy or combined with laser versus laser monotherapy for diabetic macular edema. Ophthalmology 2011;118:615-25. [CrossRef]

6. Zhioua I, Semoun O, Lalloum F, Souied EH. Intravitreal Dexamethasone Implant in Patients with Ranibizumab Persistent Diabetic Macular Edema. Retina 2015;35:1429-35. [CrossRef]

7. Sophie R, Lu N, Campochiaro PA. Predictors of Functional and Anatomic Outcomes in Patients with Diabetic Macular Edema Treated with Ranibizumab. Ophthalmology 2015;122:1395401. [CrossRef]

8. Channa R, Sophie R, Khwaja AA, Do DV, Hafiz G, Nguyen QD, et al; READ-2 Study Group. Factors affecting visual outcomes in patients with diabetic macular edema treated with ranibizumab. Eye (Lond) 2014;28:269-78. [CrossRef]

9. Maryam AK, Tafgeh M, Mahmoud M, Pasha A, Ahad S, Khalil GF. Short term effect of intravitreal bevacizumab for diabetic macular edema associated with epiretinal membrane. Rom J Ophthalmol 2018;62:212-6. [CrossRef]

10. Lai IA, Hsu WC, Yang CM, Hsieh YT. Prognostic factors of shortterm outcomes of intravitreal ranibizumab in diabetic macular edema. Int J Ophthalmol 2017;10:765-71.

11. Wong Y, Steel DHW, Habib MS, Stubbing-Moore A, Bajwa D, Avery PJ; Sunderland Eye Infirmary study group. Vitreoretinal interface abnormalities in patients treatedwith ranibizumab for diabetic macular oedema. Graefes Arch Clin Exp Ophthalmol 2017;255:733-42.

12. Yoon D, Rusu I, Barbazetto I. Reduced effect of anti-vascular endothelial growth factor agents on diabetics with vitreomacular interface abnormalities. Int Ophthalmol 2014;34:817-23.

13. Kulikov AN, Sosnovskii SV, Berezin RD, Maltsev DS, Oskanov $\mathrm{DH}$, Gribanov NA. Vitreoretinal interface abnormalities in diabetic macular edema and effectiveness of anti-VEGF therapy: an optical coherence tomography study. Clin Ophthalmol 2017;11:1995-2002. [CrossRef]

14. Ercalik NY, Imamoglu S, Kumral ET, Yenerel NM, Bardak H, Bardak Y. Influence of the epiretinal membrane on ranibizumab therapy outcomes in patients with diabetic macular edema. Arq Bras Oftalmol 2016;79:373-5. [CrossRef]

15. Cheung N, Tan SP, Lee SY, Cheung GCM, Tan G, Kumar N, et al.
Prevalence and risk factors for epiretinal membrane: the Singapore Epidemiology of Eye Disease study. Br J Ophthalmol 2017;101:371-6.

16. Ophir A, Martinez MR, Mosqueda P, Trevino A. Vitreous traction and epiretinal membranes in diabetic macular oedema using spectral-domain optical coherence tomography. Eye (Lond) 2010;24:1545-53. [CrossRef]

17. Ghazi NG, Ciralsky JB, Shah SM, Campochiaro PA, Haller JA. Optical coherence tomography findings in persistent diabetic macular edema: the vitreomacular interface. Am J Ophthalmol 2007;144:747-54. [CrossRef]

18. Mao J, Wu H, Liu C, Zhu C, Lao J, Chen Y, et al. Changes in metamorphopsia, visual acuity, and central macular thickness after epiretinal membrane surgery in four preoperative stages classified with OCT B-scan images. J Ophthalmol 2019;2019:1-8.

19. Mathew C, Yunirakasiwi A, Sanjay S. Updates in the Management of Diabetic Macular Edema. J Diabetes Res 2015;2015:18. [CrossRef]

20. Kim JY, Kim DY, Kim KT, Song J, Chae JB, Kim JG. Visual Prognostic Factors of Epiretinal Membrane Surgery in Patients with Pseudophakia. Ophthalmologica 2020;243:43-50. [CrossRef]

21. Chang CK, Cheng CK, Bai $\mathrm{CH}$, Peng $\mathrm{CH}$, Hu CC. Development of vitreomacular interface abnormality in patients with diabetic macular edema. Taiwan J Ophthalmol 2012;2:93-8.

22. Akbar Khan I, Mohamed MD, Mann SS, Hysi PG, Laidlaw DA. Prevalence of vitreomacular interface abnormalities on spectral domain optical coherence tomography of patients undergoing macular photocoagulation for centre involving diabetic macular oedema. Br J Ophthalmol 2015;99:1078 -81. [CrossRef]

23. Kawasaki R, Wang JJ, Sato H, Mitchell P, Kato T, Kawata S, et al. Prevalence and associations of epiretinal membranes in an adult Japanese population: the Funagata study. Eye (Lond) 2009;23:1045 -51. [CrossRef]

24. Namba R, Kaneko H, Suzumura A, Shimizu H, Kataoka K, Takayama K, et al. In Vitro Epiretinal Membrane Model and Antibody Permeability: Relationship With Anti-VEGF Resistance in Diabetic Macular Edema. Invest Ophthalmol Vis Sci 2019;60:2942-9. [CrossRef]

25. Cakır A, Sagdıc SK, Duzgun E, Erden B, Ayata A, Unal MH. Long-term effects of intravitreal ranibizumab treatment on retinal ganglion cell complex and choroidal thickness. New Front Ophthalmol 2018;4:1-5. [CrossRef]

26. Ünlü C, Erdogan G, Onal Gunay B, Sezgin Akcay Bl, et al. Subfoveal choroidal thickness changes after intravitreal bevacizumab therapy for neovascular age-related macular degeneration. Int J Ophthalmol 2015;8:849-51.

27. Tang F, Xu F, Zhong H, Zhao X, Lv M, Yang K, et al. Comparison of subfoveal choroidal thickness in eyes with CRVO and BRVO. BMC Ophthalmol 2019;19:133. [CrossRef] 